\title{
NANOTECHNOLOGY IN OPTICS
}

\author{
Natalia Kamanina ${ }^{1}$
}

\begin{abstract}
It is well known that the optical materials are unique and perspective. Optical materials and the devices based on them are operated in the broad spectral range: In the UV spectral range (where the wavelength $\lambda$ is approximately placed in the range of $\sim 0.1-0.4$ microns), in the VIS spectral range $(\lambda \sim 0.5-0.75$ microns), and in the IR spectral range ( $\lambda$ is larger than the 0.75-1 microns). These materials can be considered to resolve the different complicated tasks. To study optical materials different techniques and methods should be scrupulously used. Among different applied methods namely the laser oriented technique and nanostructuration approach have some unique features. It can be considered as the effective dominant approach in order to reveal the change of all basic physical-chemical characteristics of the materials. Our own steps in this direction have partially been recently shown too. In the current paper, advantages of the modification of optical material surfaces via a nanotechnology approach will be shown. The surface relief change provokes the spectral, mechanical and wetting phenomena changes. A $\mathrm{CO}_{2}$-laser is applied to modify the optical materials surfaces under the condition when the carbon nanotubes are deposited in vertical position at the materials surfaces. This process permits to organize covalent bonding between the carbon atoms and the model matrix ones. An emphasis will be given on the surface modifications of the materials, such as: $\mathrm{LiF}, \mathrm{CaF}_{2}, \mathrm{KBr}, \mathrm{BaF}_{2}, \mathrm{Sc}$, some polymer surface, etc. Mechanisms responsible for the spectral characteristics change, mechanical hardness as well as the increase of the wetting angle will be discussed. The area of the application of the materials studied can be increased.
\end{abstract}

UDC Classification: 535; 538.9; 539; 544; DOI: http://dx.doi.org/10.12955/cbup.v6.1302

Keywords: Carbon nanotubes, coatings at the interface, inorganics, reflection, laser-matter interaction

\section{Introduction}

Optical materials occupy a unique due to their strategic application in space, aircraft building, automotive, and various civil applications. Moreover optical materials are efficiently used in general optoelectronic, in laser and display techniques as well as in the biomedicine, solar energy and gas storage areas. Such materials and the devices based on the optical materials are operated in the UV spectral range, in the VIS spectral range, in the IR-spectral range and can be considered advantageous in resolving different complicated tasks. For example, $\mathrm{MgF}_{2}$ structures are used in hospitals to disinfect the premises when using ultraviolet radiation; Si-based substrates are considered effective matrices for solar energy elements; $\alpha$-Si:H systems are applied as effective photosensitive layers in electrically- and light-addressed spatial light modulators; $\mathrm{ZnO}$ and ITO conducting layers are used to apply the bias voltage for display and the solar energy constructions; $\mathrm{KBr}$ and $\mathrm{LiF}$ crystals are considered important materials for Furrier spectrometry and the passive shutter. It should be noted that the traditional optical materials include inorganic and organic compounds in which classical optical and the nonlinear optical effects can be activated, which has been scrupulous shown in the classical books (Landsberg, 1976, Feynman, 1976, Sze, 1981, Arakelyan, 1984, Vasilev, 1987, Born, 1999, Gutman, 1967, Akhmanov, 1997, Yariv, 2007, Agrawal, 2008).

It should be mentioned that in the past optical materials were divided according to their electroconducting properties, taking into account the changes in the dielectric constant and charge carriers' mobility (Gutman, 1967). However now, because of the possibility of fairly easy modifications of physical-chemical parameters of optical matrices with a nanostructuring process, the priority of such a classification is given to spectral, mechanical, laser and photorefractive characteristics (Koch, 2002, Valiev, 2013, Kamanina, 2011, 2012, 2014). Indeed, the different types of classical methods in addition to new innovative approaches can be used to modify the interfaces of optical materials. The surface modification provokes dramatical changes in optical properties via their nanostructuring by carbon nanotubes (CNTs). The advantage of this nanostructuration process use is connected with the fact that the covalent bonding can be developed between matrix materials and CNTs that leads to change the transmission, scattering, reflection, absorption, refraction, etc. Using a structuration approach the spectral, refractive, mechanical properties, wetting phenomena, refractivity can be essentially changed. Some evidence of the increase of the transparency of the inorganic materials and their mechanical characteristics has been recently shown in the papers by Kamanina (2017, 2017).

The main idea of the improvement of the properties of some group of optical materials is regarded to the evidence that the refractive index of the CNTs $(n \sim 1,05-1,1)$ is substantially less than the refractive

\footnotetext{
${ }^{1}$ Vavilov State Optical Institute, St.- Petersburg, Russia; St.-Petersburg Electrotechnical University ("LETI”), St.-Petersburg, Russia, nvkamanina@mail.ru
} 
index of the treated materials, and the diameter of the CNTs allows one to embed these nanoobjects in the voids of the lattice of matrix systems. This contributes to the homogenization of interfaces with very small (air) and relatively large (matrix materials, in which surfaces the CNTs are incorporated) refractive indexes, which causes a change in the range of transmission, at least. In the current paper the property modifications of the materials, which surfaces are structured by the laser oriented deposition (LOD) method are shown. The spectral, mechanical properties, as well as the wetting features of the materials are discussed. Moreover, the change of the wetting angle for some polymer structures is presented.

\section{Experimental conditions, materials and results}

It should be mentioned that to modify the properties of the inorganic materials via their surface treatment, the CNTs were deposited using IR $\mathrm{CO}_{2}$-laser with $p$-polarized irradiation at the wavelength of $10.6 \mu \mathrm{m}$ and a power of $30 \mathrm{~W}$. Moreover, when SWCNTs were placed at the materials interface, an electric field of $100-600 \mathrm{~V} \times \mathrm{cm}^{-1}$ has been applied in order to orient the nanotubes during the deposition. The spectra of the nanotubes-treated materials were obtained using VIS and Furrier FSM1202 devices. The microhardness was obtained via a PMT-3M instrument ("LOMO", SaintPetersburg). A special accent was given to observe the relief at the material surface via the checking of the wetting angle. A OCA 15EC device purchased from LabTech Co. (Saint-Petersburg-Moscow, Russia) was applied in this case.

\subsection{LiF materials spectra, hardness and wetting angle}

It is well known that the lithium fluoride LiF material is a unique colorless cubic optical crystal with the unit cell parameter close to $0,40279 \mathrm{~nm}$. It has a very high transparency from 0.12 to 6 microns, therefore when it is used in a vacuum, it is used in the ultraviolet region of the spectrum as well as in the IR-spectral range. It is often transparent in the indicated spectral regions and has higher optical transparency than other crystals. The refractive index $\mathrm{n}$ of LiF materials can be changed with a change in the wavelength as followings: 1,77 (at 112,7 nm), 1,3978 (at $420 \mathrm{~nm}$ ), 1,3915 (at $620 \mathrm{~nm}$ ), 1,2912 (at $6200 \mathrm{~nm}$ ) (Lide, 2009). The structuration of the LiF surface permits it to change its basic parameters. The spectral feature modifications are shown in Fig.1, $a, b$.

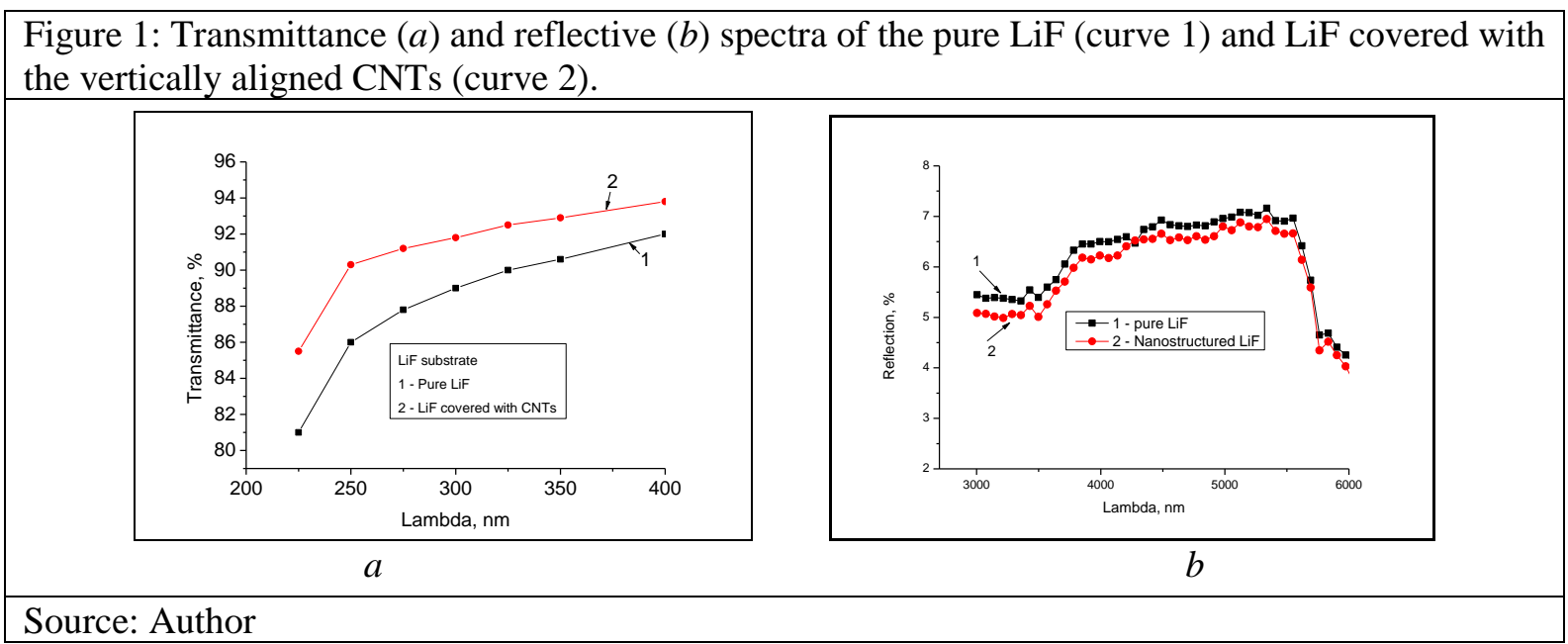

Analyzing the spectral dependence, it can be seen that the transparency and reflective characteristics are occur at the same time as each other and the Fresnel losses (connected with the relation of the refractive index between CNTs and matrix material one) from the nanostructures surfaces can be decreased by using the CNTs laser oriented deposition technique. Moreover, the wetting angle and the mechanical hardness can be changed successfully as well. The change of the mechanical characteristics is presented in Table 1. Considering the data shown in Table 1, one can see that the increase in the microhardness was determined by the value of $10 \%$ after the procedure to treat the material surfaces with CNTs with high Young's module (some TeraPa) using the laser oriented deposition technique.

The increase of the LiF material wetting angle after the LOD technique is shown in Fig.2. One can see that the wetting angle has been increased from 97,5-97,8 degrees to 107,3-107,5 degrees. Moreover, 
the wetting angle has been obtained only after the treatment with CNTs on one side of the LiF substrate. Thus, the surface modification can cause better results after a two sided treatment.

\begin{tabular}{|c|c|c|c|}
\hline & Materials & Micro-hardness, $\mathrm{Pa}$ & $\begin{array}{c}\text { Increasing coefficient of the microhardness } \\
\text { change, } \%\end{array}$ \\
\hline & $\mathrm{LiF}$ & $1.304 \times 10^{9}$ & \multirow[t]{2}{*}{ 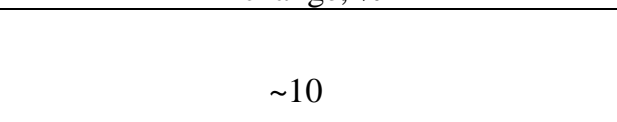 } \\
\hline & $\mathrm{LiF}+\mathrm{CNTs}$ & $1.442 \times 10^{9}$ & \\
\hline
\end{tabular}

Figure 2: The value of the wetting angle of the LiF crystal before $(a)$ and after $(b)$ its surface nanostructuring

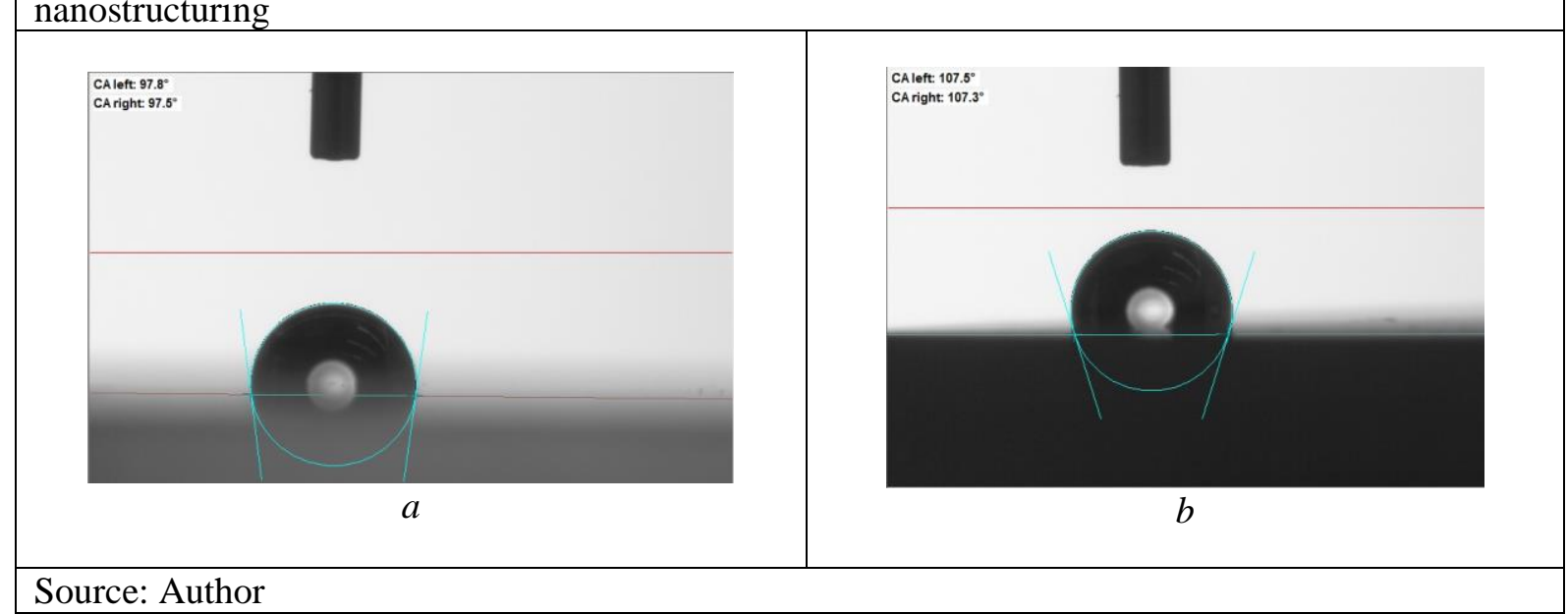

\section{2. $\mathrm{CaF}_{2}$ materials spectra, hardness and wetting angle}

Modification of the properties of the $\mathrm{CaF}_{2}$ structures by the LOD technique predicts a dramatic change in the basic parameters. The spectral dependences are shown in Fig.3.

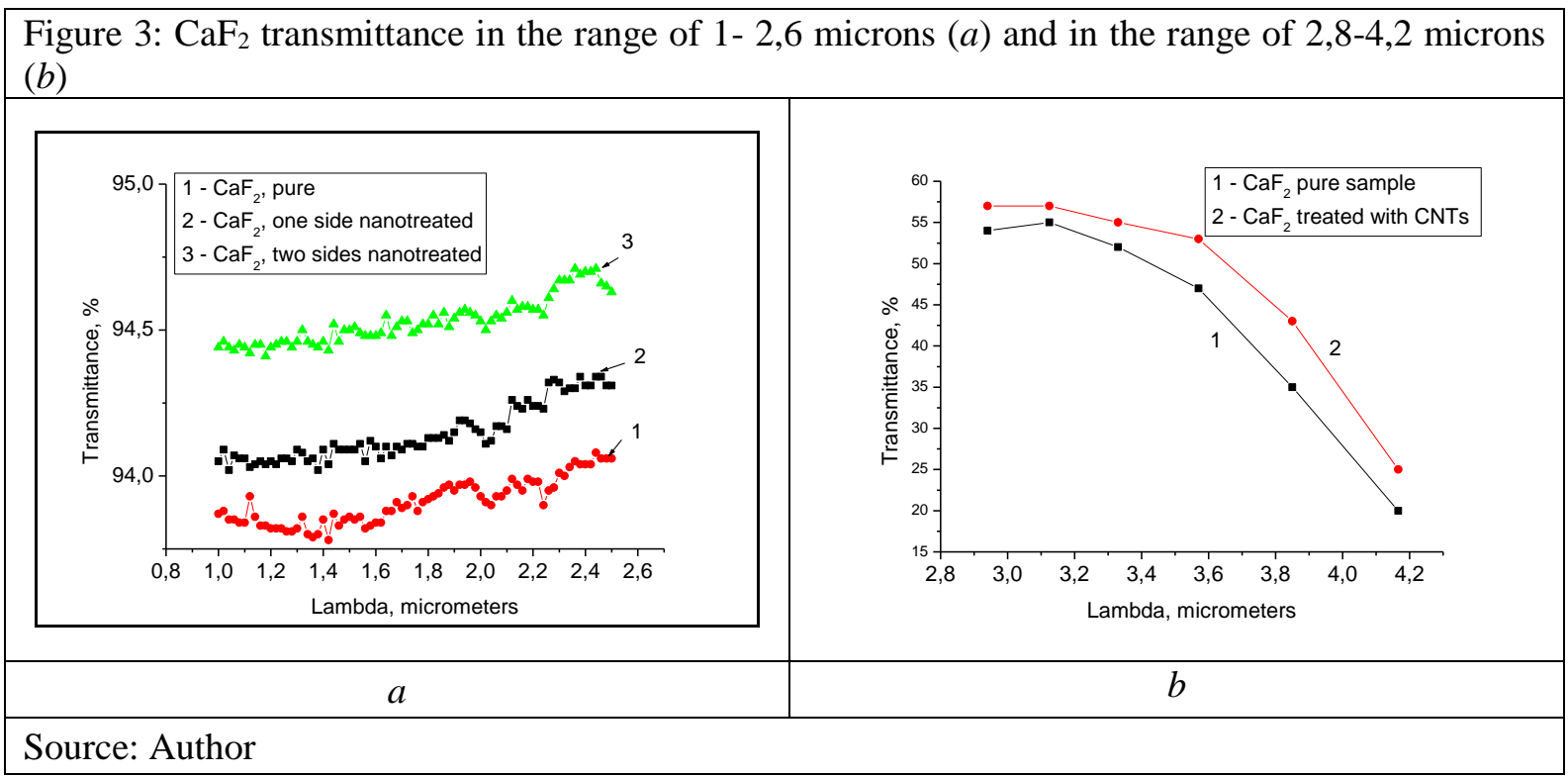

It should be mentioned that calcium fluoride $\mathrm{CaF}_{2}$ materials are widely used in laboratory research and industry. Calcium fluoride is a component of special glasses, enamels, ceramics, optical and laser materials. Calcium fluoride is applied as optical material for the infrared and the ultraviolet spectral range, and it can be used as a component in the lenses that reduces the light scattering as well. Moreover, this material has used in polarization optics (Rauch, 1973). 
Analyzing the data shown in Fig.3, one can see the transmittance change in the near and middle IRrange under the LOD treatment. Fig.3, $a$ shows the data on the transmission of the calcium fluoride samples with treatments of one and two surfaces; Fig.3, $b$ shows the results of changes in the transmission in the treatment of only one surface. Therefore, one can expect the better influence of the CNTs structuration on the spectra when the $\mathrm{CaF}_{2}$ substrate has been treated from two sides. Moreover, analyzing the reflective features studied at the same wavelengths one can see the decrease of the Fresnel loses are up to 2-3\%. These spectral dependences are in good coinciding with the microhardness improvement, which is shown in Table 2. Moreover, the experiments with the checking of the wetting angle have been made for the pure and for the nanostructured $\mathrm{CaF}_{2}$ materials. The wetting angle has been increased from 81,9-82,6 degrees up to 96,6-97,1 degrees after the CNTs oriented laser deposition procedure application.

\begin{tabular}{|l|c|c|c|c|}
\hline \multicolumn{3}{|c|}{ Table 2: Micro-hardness of the pure and CNTs treated $\mathrm{CaF}_{2}$ at the indenter force $\sim 40 \mathrm{~g}$} \\
\hline \multirow{4}{*}{} & Materials & Micro-hardness, $\mathrm{Pa}$ & $\begin{array}{c}\text { Increasing coefficient of the microhardness } \\
\text { change, } \%\end{array}$ & \\
\cline { 2 - 3 } & $\mathrm{CaF}_{2}$ & $0.181 \times 10^{9}$ & $\sim 10$ \\
\cline { 2 - 3 } & $0.199 \times 10^{9}$ & $\mathrm{CaF}_{2}+\mathrm{CNTs}$ & \\
\hline
\end{tabular}

Indeed, other different inorganic crystals used in the general optoelectronic area can be modified via the surface structuration in order to reveal the change of their spectral and mechanical characteristics and the wetting properties as well. Some comparative results are shown in Table 3. From data of the micro-hardness and wetting angle change of the pure and the structured inorganic crystals shown in Table 3, it should be remarked a significant change of these parameters. Thus, using the consideration based on the $\mathrm{LiF}, \mathrm{CaF}_{2}, \mathrm{KBr}, \mathrm{BaF}_{2}$ inorganic crystals it can be proposed that using the CNTs via a LOD application is an innovative way to modify the physical-chemical characteristics of this type of inorganic materials.

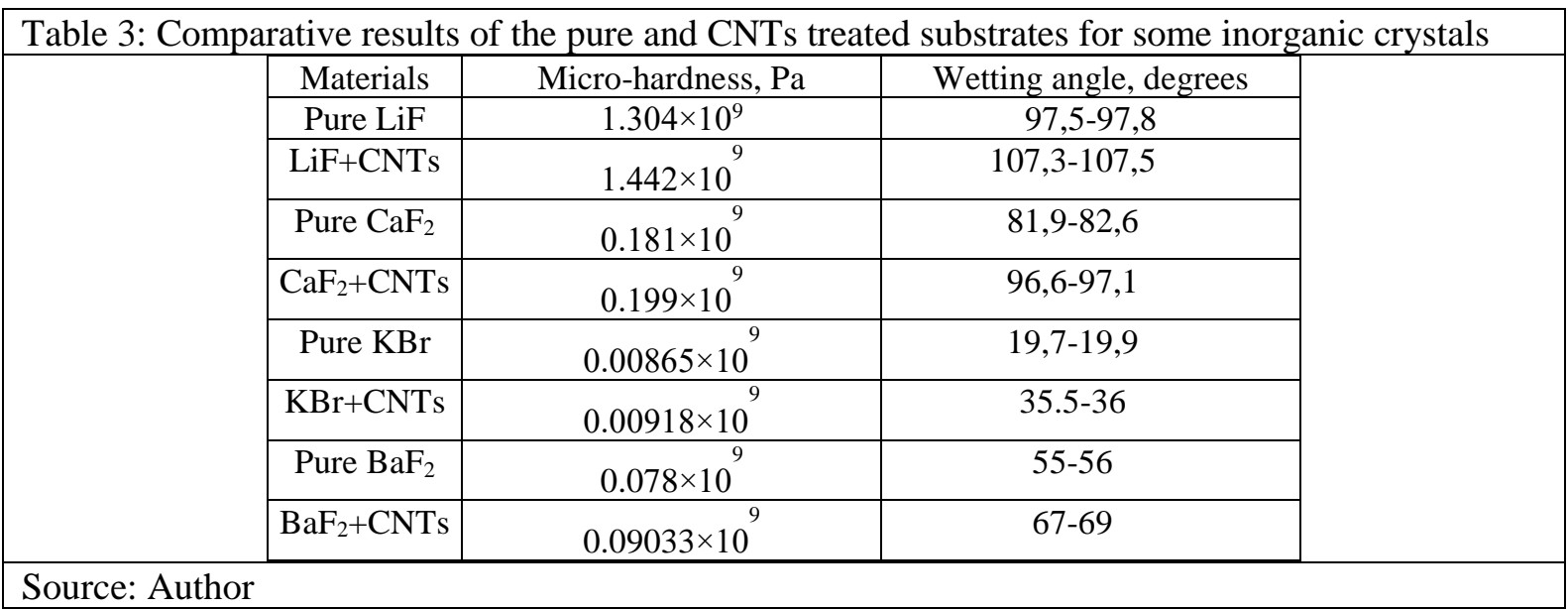

\subsection{Sc materials spectral parameters modifications}

It is well known that scandium Sc is a light metal, which is currently widely used in general optoelectronics and in laser technology. Moreover, it can be used as solar cells elements. The last application requires modifying the Sc properties by using CNTs in order to increase the charge carrier mobility and to obtain functional diode parameters (Leijing Yang, 2011, 2012, Qingsheng Zeng, 2012). Our steps in this direction have shown that the CNTs can organize the covalent bonding with the surface Sc atoms. The simulation and experimental results are good coincided. It should be noted that the Sc \#40229 from Alfa Aesar was used for this study. Investigation of the penetration process of the CNT in the substrate were performed using the classical molecular dynamics simulations, implemented in the LAMMPS software (Plimpton, 1995). The Sc substrate with low indexes surfaces $\mathrm{Sc}(0001)$ was considered for the penetration process. It should be remarked that the Sc substrate consisted of $\sim 45000$ atoms. Behavior of the Sc substrate under the temperature conditions was described within the framework of the method shown in Daw (1983) with the fitted parameters as 
shown in Baskes (1994). To describe the interaction between CNT and the Sc substrate the 12/6 Lennard-Jones potential $E=4 \varepsilon\left[\left(\frac{\sigma}{r}\right)^{12}-\left(\frac{\sigma}{r}\right)^{6}\right]$ was fitted with the interaction parameters: $\varepsilon=0.011 \mathrm{eV}$, $\sigma=1.8 \AA$. The many-body Tersoff approach was used (Tersoff, 1989). The velocity of the CNT (diameter was from $0.64 \mathrm{~nm}$ to $3.35 \mathrm{~nm}$ ) was varied from $100 \mathrm{~m} / \mathrm{s}$ to $600 \mathrm{~m} / \mathrm{s}$. Analyzing the calculated data one can see that increasing the CNTs diameter leads to a decrease in penetration depth. This calculation is connected with the experimentally obtained reflection spectra of the Sc materials placed on the Si-substrate. Fig.4 presents the data. It should be noted that the spectral parameters of the Si substrate with the CNTs modification have been shown in the paper Kamanina (2017); the molecular dynamic simulation for the Si-CNTs surfaces was previously done as well.

Figure 4: Reflection of the Sc-Si-substrate: pure Si (black curve) and Si covered with Sc coating (red curve) placed on the polished Si substrate

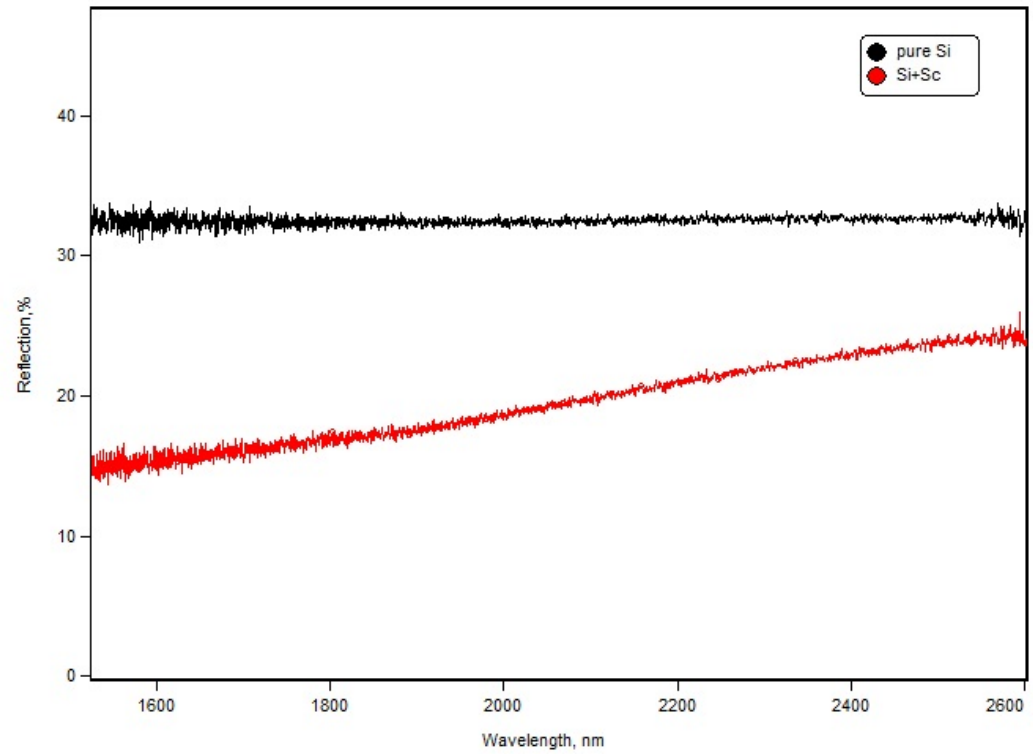

\section{Source: Author}

One can see from Fig.4 that the reflection of the compounds based on the Sc-Si-systems decreased dramatically. This fact is connected with the relation between refractive indexes of the $\mathrm{Si}(\sim 3.4)$ and Sc ( 1.7-1.9) materials and connected with the classical relation: $n_{\text {coating }} \approx \sqrt{ } n_{\text {substrate. This }}$ relation between the refractive indexes explains a larger absorption of the Si matrix used in solar cells elements when this Si matrix can be modified with $\mathrm{Sc}$ or Sc+CNTs coatings. Therefore, scandium can be considered, along with CNTs, as an effective coating for silicon substrates.

\subsection{Polymers surface features revealed via structuration}

In addition, some interesting results, which support the mentioned above influence of the surface structuration on the wetting angle change, can be connected with the influence of the sensitization of the body of the polymer materials on the wetting angle as well. The polyimide structures can be considered to indicate these results. The data is presented in Fig. 5.

Fullerenes $\mathrm{C}_{70}$ were used in this case. One can see that the wetting angle can be changed from 73 degrees up to 103 degrees when the content of the fullerene $\mathrm{C}_{70}$ in the polyimide matrix was varied from 0 to $1,0 \mathrm{wt} . \%$. It should be mentioned that before such polyimides are widely used as the photosensitive structures in the solar energy and nonlinear optics. We have used the polyimide photosensitive layers in the spatial light modulators and as the optical limiting (OL) construction as well (Kamanina, 1997, 1999, 2002). As the OL mechanisms we have considered the reverse saturation absorption, intermolecular charge transfer complex formation and the change of the energy losses via diffraction. The current results, namely shown in Fig.5, can include some new mechanism for the explanation of optical limiting effect. The evidence of the influence of the polymer body provokes the 
development of some relief at the polymer surface that can decrease the light propagation due to scattering from the structured surfaces. Thus, the polymers with structured surfaces can be considered as separate elements, which do not require being pressed into triacetate cellulose, or be placed between glass plates.

Figure 5: The wetting angle of the studied organics based on polyimide when the content of the fullerene $\mathrm{C}_{70}$ inside the matrix body has been varied: $0 \%(a)$; and 1,0 wt. $\%(b)$

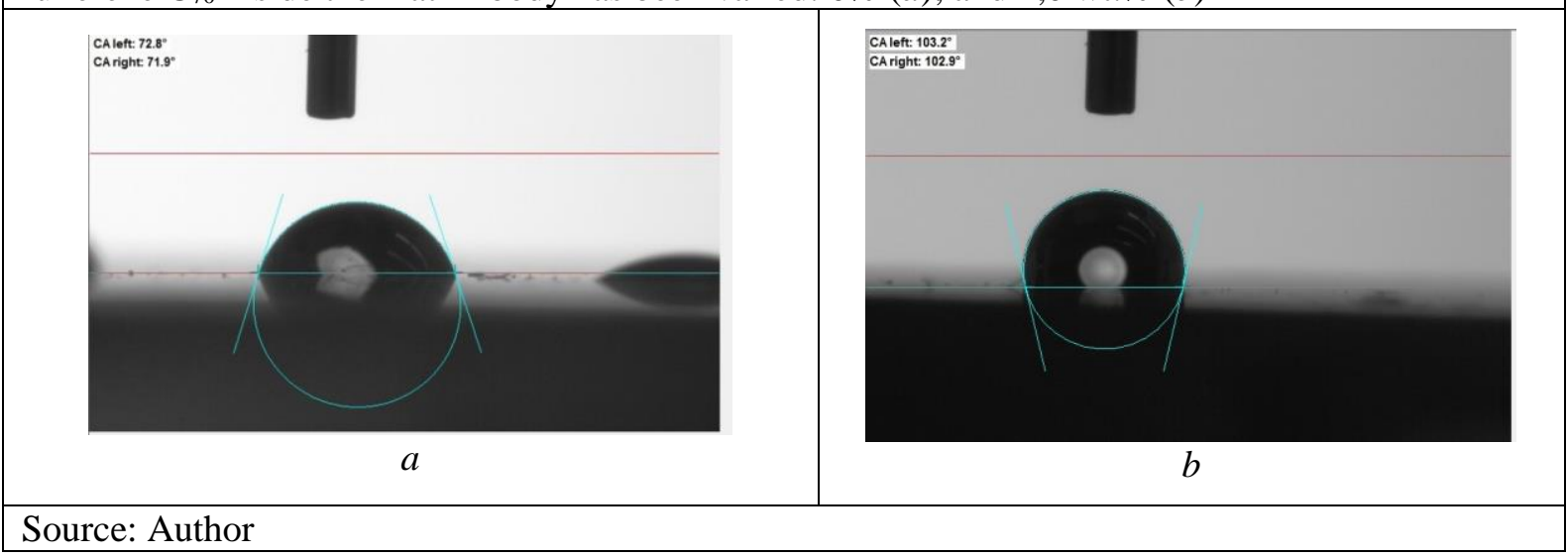

\section{Conclusion}

To summarize the results one can conclude that: 1). Structuration of the inorganic material surfaces (based, for example, on the $\mathrm{LiF}$ and $\mathrm{CaF}_{2}$ structures) has predicted the change in spectral, mechanical and wetting features. The increase of the transparency, hardness, and wetting angle has also been revealed. 2). Structuration of the organic material body (polymers) can cause a change in their surface features. It leads to the increase of the wetting angle dramatically. It permits to use of structured polymers as separate elements in the different complex optoelectronic schemes. 3). Area of applications of the materials whose properties can be modified via the structuration process with effective nanoparticles can be extended essentially.

\section{Acknowledges}

The obtained results have been partially supported by RFBR grant \#13-03-00044 (2013-2015), project "Nanocoating-GOI" (2012-2015), international FP7 Program, Marie Curie Action, Project "BIOMOLEC" (2011-2015), Project "Adaptatsiya" under the Russia-Israel collaboration agreement (2017). Author wishes to thank their colleagues Dmitry Kvashnin (Emanuel Institute of biochemical physics RAS, Institute of Science and Technology "MISiS") and Pavel Sorokin (Technological Institute for Superhard and Novel Carbon Materialstheir, Institute of Science and Technology "MISiS") for the help with the quantum chemical calculations.

\section{References}

Agrawal, G. Applications of Nonlinear Fiber Optics. Academic Press, 2008, 508 p.

Akhmanov, S.A. \& Nikitin, S.Yu. Physical optics, Oxford Press. Oxford, 1997. 656 p.

Arakelyan, S.M. \& Chilingaryan, Yu.S. Nonlinear Optics of Liquid Crystals, Moscow: Nauka, 1984, 360 p.

Baskes, M.I. \& Johnson, R.A. (1994). Modified embedded atom potentials for HCP metals. Model. Simul. Mater. Sci. Eng. 2(1), 147-163.

Born, M. \& Wolf, E. Principles of Optics: Electromagnetic Theory of Propagation, Interference and Diffraction of Light', Cambridge University Press, 1999. 987 p.

Daw, M.S. \& Baskes, M.I. (1983). Semiempirical, Quantum Mechanical Calculation of Hydrogen Embrittlement in Metals'. Phys. Rev. Lett. 50(17), 1285-1288.

Feynman, R.Ph., Lejton, R., \& Sends, M., Feynman's physical lectures. Moscow: Mir, Books seria, Vol.7, 1976, 288 p.

Gutman, F. \& Lyons, L.E. Organic Semiconductors, New York: J. Wiley \& Sons, 1967, 858 p.

Kamanina N.V. \& Vasilenko N.A. (1997). Influence of operating conditions and of interface properties on dynamic characteristics of liquid-crystal spatial light modulators. Opt. Quantum Electron. 29(1), pp. 1-9. Kamanina N.V. (1999). Reverse saturable absorption in fullerene-containing polyimides. Applicability of the Förster model. Opt. Commun. 162(46), pp.228-232. DOI: 10.1016/S0030-4018(99)00095-4.

Kamanina N.V. (2002). Mechanisms of optical limiting in $\pi$-conjugated organic system: fullerene-doped polyimide. Synthetic Metals. 127(1-3), pp.121-128. DOI: 10.1016/S0379-6779(01)00598-7. 
Kamanina, N.V. (2011). Features of liquid crystal display materials and processes, Edited by InTech, Croatia. 2011. 240 p. First published November, 2011, Published by InTech, Janeza Trdine 9, 51000 Rijeka, Croatia. ISBN 978-953-307-899-1.

Kamanina, N.V. (2012). Nonlinear Optics, InTech, Croatia. 2012. 224 p. First published February, 2012. Published by InTech, Janeza Trdine 9, 51000 Rijeka, Croatia. ISBN 978-953-51-0131-4

Kamanina, N.V. (2014). Features of Optical Materials Modified with Effective Nanoobjects: Bulk Properties and Interface", New York, Physics Research and Technology, "Novinka", Published by Nova Science Publishers, Inc., New York, 2014, 116 p. ISBN: 978-1-62948-033-6

Kamanina, N.V. (2017). Carbon structures as effective modifiers of the materials' basic properties, Proceed. of CBU International conference on innovations in science and education, March 22-24, 2017, PRAGUE, CZECH REPUBLIC, Vol.5, p.1135-1142, 2017. www.cbuni.cz, www.journals.cz.

Kamanina, N.V. (2017). Chapter 8. Perspective of the Structuration Process Use in the Optoelectronics, Solar Energy, and Biomedicine, p.167-183, 2017. http://dx.doi.org/10.5772/68123 in the book "Nanomechanics" Edited by Alexander Vakhrushev, ISBN 978-953-51-3182-3, 192 pages, 2017. DOI: 10.5772/65466

Koch, C.C. Nanostructured Materials: Processing, Properties and Applications, Taylor \& Francis, 2002. 176 p. ISBN 0815514514,9780815514510

Landsberg, G.S., Optica. Moscow: Nauka, 1976 and 2003, 848 p.

Leijing, Yang, Sheng, Wang, Qingsheng, Zeng, Zhiyong, Zhang, Tian, Pei, Yan, Li. \& Lian-Mao, Peng. (2011). Efficient photovoltage multiplication in carbon nanotubes. Nature Photonics. 5, 672-676. doi:10.1038/nphoton.2011.250

Leijing, Yang, Sheng, Wang, Qingsheng, Zeng, Zhiyong, Zhang, Yan, Li, Weiwei, Zhou, Jie, Liu, Lian-Mao, Peng. (2012). Channel-Length-Dependent Transport and Photovoltaic Characteristics of Carbon-Nanotube-Based, Barrier-Free Bipolar Diode. ACS Appl. Mater. Interfaces. 4(3), 1154-1157. DOI: 10.1021/am201778x

Lide, D. R. (Ed.), (2009). "CRC Handbook of Chemistry and Physics", 90th edition. CRC Press; Taylor and Francis, 2009. 2828 p. ISBN 1420090844.

Plimpton,S. (1995). Fast Parallel Algorithms for Short-Range Molecular Dynamics. J.Comput.Phys.117(1),1-19.

Qingsheng, Zeng, Sheng, Wang, Leijing, Yang, Zhenxing, Wang, Tian, Pei, Zhiyong, Zhang, Lian-Mao, Peng, Weiwe,i Zhou, Jie, Liu, Weiya, Zhou. \& Sishen, Xie (2012). Carbon nanotube arrays based high-performance infrared photodetector. Optical Materials Express. 2(6), 839-848. DOI: 10.1364/OME.2.000839

Rauch, R. (1973). Photoluminescence of color centers in crystals of alkaline earth fluorides. News of Academy of Sciences of the USSR. Series Physical, 37(3), 595-598.

Sze, S. M. Physics of Semiconductor Devices, Second Edition. A Wiley-Interscience publication. John Wiley \& Sons New York-Toronto-Singapore, 1981, $456 \mathrm{p}$.

Tersoff, J. (1989). Modeling solid-state chemistry: Interatomic potentials for multicomponent systems. Phys. Rev. B. 39(8), $5566-5568$

Valiev, R.Z., Zhilyaev, A.P. \& Langdon, T.G. Bulk Nanostructured Materials: Fundamentals and Applications, 456 pages. December 2013. Wiley.com. ISBN: 978-1-118-09540-9

Vasilev, A.A., Casasent, D., Kompanets, I.N., \& Parfenov, A.V. Spatial Light Modulators, Radio i Svyaz', Moscow, 1987 (in Russian), 320 p.

Yariv, A. \& Yeh P. Photonics: optical electronics in modern communications, 6th. Oxford University Press, 2007. ISBN 9780-19-517946-0. 